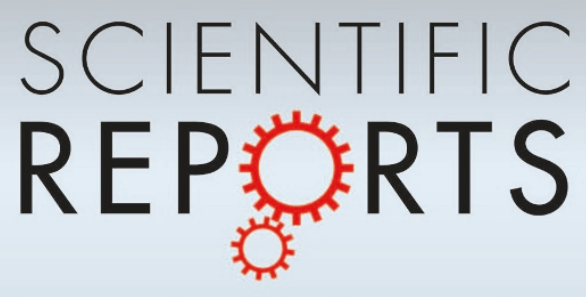

OPEN

SUBJECT AREAS:

ELECTRONIC PROPERTIES

AND DEVICES

SURFACES, INTERFACES AND

THIN FILMS

MATTER WAVES AND PARTICLE

BEAMS

ELECTRONIC AND SPINTRONIC DEVICES

Received

16 October 2014

Accepted

19 January 2015

Published

13 February 2015

Correspondence and requests for materials should be addressed to J.D.W. (jwalls@miami. edu)

\section{Suppressing Klein tunneling in graphene using a one-dimensional array of localized scatterers}

\author{
Jamie D. Walls \& Daniel Hadad \\ Department of Chemistry, University of Miami, Coral Gables, Florida 33124, USA.
}

Graphene's unique physical and chemical properties make it an attractive platform for use in micro- and nanoelectronic devices. However, electrostatically controlling the flow of electrons in graphene can be challenging as a result of Klein tunneling, where electrons normally incident to a one-dimensional potential barrier of height $V$ are perfectly transmitted even as $V \rightarrow \infty$. In this study, theoretical and numerical calculations predict that the transmission probability for an electron wave normally incident to a one-dimensional array of localized scatterers can be significantly less than unity when the electron wavelength is smaller than the spacing between scatterers. In effect, placing periodic openings throughout a potential barrier can, somewhat counterintuitively, decrease transmission in graphene. Our results suggest that electrostatic potentials with spatial variations on the order of the electron wavelength can suppress Klein tunneling and could find applications in developing graphene electronic devices.

S ince graphene's initial discovery ${ }^{1}$, much research has been undertaken into studying graphene's unique physical and chemical properties ${ }^{2,3}$, which are a consequence of its two-dimensional structure. Graphene consists of carbon atoms arranged in a honeycomb lattice made up of two trigonal sublattices that each contribute a carbon atom to the unit cell, thereby imparting a pseudospin character to the electrons in graphene. From tight-binding calculations ${ }^{4}$, the quasiparticle spectrum of graphene is linearly proportional to the magnitude of the wave vector, $|\vec{k}|$, when expanded about two distinct wave vectors, $\pm \vec{K}= \pm \frac{4 \pi \sqrt{3}}{9 b} \hat{x}$ with $b=1.42 \AA$ being the $\mathrm{C}$-C bond length. The wave vectors $\pm \vec{K}$ are referred to as Dirac points due to the similarity of the electronic spectrum in graphene to that of a massless two-dimensional Dirac fermion ${ }^{5}$. A consequence of the linear dispersion and the pseudospin nature of electron waves in graphene is Klein tunneling ${ }^{6}$, where massless Dirac fermions normally incident to a potential step barrier are not reflected even when the potential barrier height becomes infinite [Figure 1(A)]. Klein tunneling makes it difficult to stop the flow or transmission of electrons electrostatically, which poses a significant challenge for incorporating graphene into new electronic devices.

One proposed method for controlling and modifying the electronic properties in graphene has been to use superlattice potentials, $\hat{V}(x, y)$. In this case, graphene's effective Hamiltonian, when expanded about the $\pm \vec{K}$ Dirac points, is given by? $^{7}$ :

$$
\hat{H}_{ \pm \vec{K}}= \pm v_{F}\left(\hat{\sigma}_{X} \hat{p}_{X}+\hat{\sigma}_{Y} \hat{p}_{Y}\right)+\hat{V}(x, y)
$$

where $\hat{\sigma}_{X}$ and $\hat{\sigma}_{Y}$ are pauli spin matrices, $\hat{p}_{X}$ and $\hat{p}_{Y}$ are momentum operators, and $\hbar v_{F}=1.0558 \times 10^{-28} \mathrm{~J}$-m. In writing Eq. (1), the spatial variations of $\hat{V}(x, y)$ are assumed to be on length scales much greater than the C-C bond length. Such superlattice potentials can, in principle, be patterned on graphene using either localized chemical modifications $s^{8,9}$ or by locally manipulating the voltages of metallic islands or electrodes ${ }^{10,11}$ placed on top of graphene. Previous theoretical work ${ }^{12-17}$ has mainly focused on using periodic potentials along a single dimension, e.g., a periodic array of square potential barriers like the one shown in Fig. 1(A). For such a Kronig-Penney potential, there is no suppression of Klein tunneling for electrons at normal incidence. Other types of superlattice potentials, such as the muffin-tin superlattice potential ${ }^{12,18}$, which consists of a two-dimensional array of cylindrically symmetric step potentials, have been theoretically shown to generate electron localization and significantly alter graphene's dispersion relationship although the transport properties through such superlattice potentials have not been examined. 


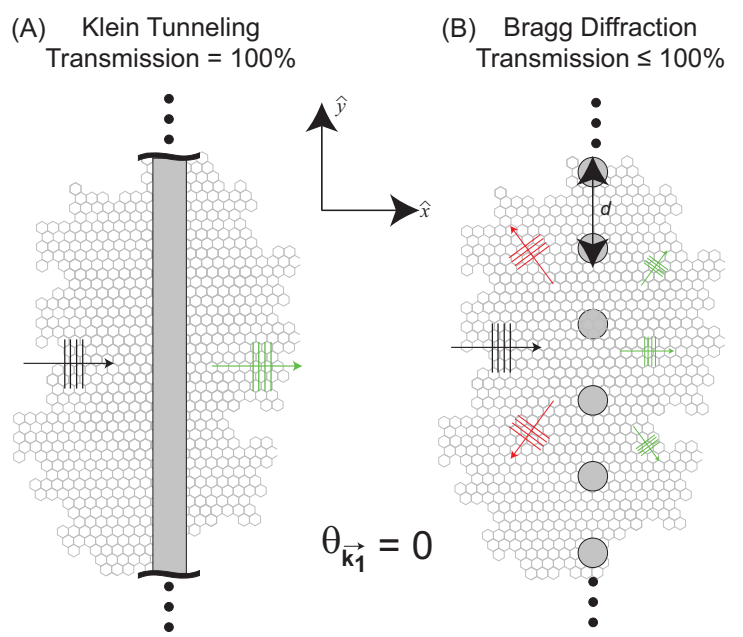

Figure $1 \mid$ Scattering of Dirac plane wave spinors at normal incidence $\left.\boldsymbol{\theta}_{\vec{k}_{1}}=0\right]$ to either (A) a one-dimensional potential barrier or (B) a onedimensional array of localized cylindrically symmetric scatterers in graphene where the $n^{\text {th }}$ scatterer is centered at $\vec{r}_{n}=n d \hat{y}$ with $d$ being the spacing between nearest neighbor scatterers. (A) For a one-dimensional barrier, a normally incident wave is perfectly transmitted as a result of Klein tunneling ${ }^{19}$. (B) For a one-dimensional array of localized scatterers, a normally incident wave can be backscattered into other open scattering channels when $\frac{d}{\lambda} \geq 1$ thereby leading to transmission probabilities that can be considerably less than unity.

For single or multiple square potential barriers, Klein tunneling is still not suppressed since normally incident waves are only allowed to undergo direct backscattering (specular reflection) from such potentials, which is prohibited due to time-reversal symmetry in graphene. Therefore, to suppress Klein tunneling, electrostatic potentials are required that generate non-specular or diffuse reflection, which is not prohibited by time-reversal symmetry. One such potential that can exhibit diffuse reflection is a one-dimensional periodic array of localized scattering potentials as shown in Figure 1(B). Such potentials appear often in optics and in atomic/matter wave diffraction experiments and can be patterned on graphene'. As shown in this work, when the electron's wavelength, $\lambda$, becomes comparable to the spacing between scatterers, $d$, non-specular reflection can lead to a dramatic reduction in the transmission through such potentials even at normal incidence. As a result, Klein tunneling in graphene can be suppressed when using a periodic array of localized scatterers.

\section{Results}

In the absence of an electrostatic potential $[\hat{V}(x, y)=0]$, the positive energy solutions to Eq. (1) with energy $E=\hbar v_{F} k_{1}=\frac{h v_{F}}{\lambda} \geq 0$ and normalized to unit flux along the $\hat{x}$-direction are the Dirac plane wave spinors $\phi_{i n c}^{\vec{K}}(\vec{r})=\sqrt{\frac{k_{1}}{2 v_{F} k_{X 1}} e^{\vec{k}_{1} \cdot \vec{r}}}\left(\begin{array}{c}1 \\ e^{i \theta_{\vec{k}_{1}}}\end{array}\right)_{\vec{K}}$ and $\phi_{i n c}^{-\vec{K}}(\vec{r})=\sqrt{\frac{k_{1}}{2 v_{F} k_{X 1}} e^{\vec{k}_{1} \cdot \vec{r}}}$ $\left(\begin{array}{c}1 \\ -e^{i \vec{k}_{1}}\end{array}\right)_{-\vec{K}}$ with wave vector $\vec{k}_{1}=k_{1}\left[\cos \left(\theta_{\vec{k}_{1}}\right) \hat{x}+\sin \left(\theta_{\vec{k}_{1}}\right) \hat{y}\right] \equiv$ $k_{X 1} \hat{x}+k_{Y 1} \hat{y}$, wavelength $\lambda=\frac{2 \pi}{k_{1}}$, and $\theta_{\vec{k}_{1}} \in\left[-\frac{\pi}{2}, \frac{\pi}{2}\right]$. Consider a Dirac plane wave spinor $\phi_{i n c}^{ \pm \vec{K}}(\vec{r})$ incident to an array of localized cylindrically symmetric scattering potentials as depicted in Fig. 2(A). Each scatterer is modeled by a simple step potential with an effective radius of $r_{s}$ so that the potential for the $n^{t h}$ scatterer is given by $V_{0} \Theta\left(\vec{r}-\vec{r}_{n}\right)$ where $\Theta\left(\vec{r}-\vec{r}_{n}\right)=1$ if $\left|\vec{r}-\vec{r}_{n}\right| \leq r_{s}$ and $\Theta\left(\vec{r}-\vec{r}_{n}\right)=0$ for $\left|\vec{r}-\vec{r}_{n}\right|>r_{s}$. To consider only intravalley scattering $\left[\vec{k} \pm \vec{K} \rightarrow \vec{k}^{\prime} \pm \vec{K}\right]$ and to neglect intervalley scattering $\left[\vec{k} \pm \vec{K} \nrightarrow \vec{k}^{\prime} \mp \vec{K}\right], r_{s}$ must be greater than the C-C bond length in graphene, i.e., $r_{s} \gg 1.42 \mathrm{~A}$.

As derived in Supporting Information, the transmitted wave function $[x \gg d], \psi_{T}^{+\vec{K}}(\vec{r})$, can be written as a sum of Dirac plane wave spinors with wave vectors along the Bragg directions, $\vec{k}_{1}^{(n)}=k_{Y 1}^{(n)} \hat{y}+k_{X 1}^{(n)} \hat{x}$ for integer $n$ where $k_{Y 1}^{(n)}=k_{Y 1}+\frac{2 \pi n}{d}$ and $k_{X 1}^{(n)}=\sqrt{k_{1}^{2}-\left(k_{Y 1}^{(n)}\right)}$ for $k_{1} \geq k_{Y 1}^{(n)}$. In this case, the transmitted wave function through the one-dimensional array of localized scatterers can be written as a sum over Dirac plane wave spinors propagating along the Bragg directions for $x \gg d$ as:

$$
\psi_{T}^{ \pm \vec{K}}(\vec{r})=\sum_{n \in \mathcal{N}} T_{n} e^{i\left(k_{Y 1}^{(n)} y+k_{X 1}^{(n)} x\right)} \sqrt{\frac{k_{1}}{2 v_{F} k_{X 1}^{(n)}}}\left(\begin{array}{c}
1 \\
\pm e^{i \theta_{\vec{k}_{1}^{(n)}}}
\end{array}\right)_{ \pm \vec{K}}
$$

where $T_{n}$ is the transmission coefficient for the $n^{\text {th }}$ Bragg direction or open scattering channel. The sum in Eq. (2) is over all open scattering channels, $\quad n \in \mathcal{N}=\left[\mathcal{N}_{\min }, \mathcal{N}_{\max }\right]$ where $\mathcal{N}_{\min }=$ $\left\{\frac{-\left(k_{1}+k_{Y 1}\right) d}{2 \pi}\right\}_{+}$and $\mathcal{N}_{\max }=\left\{\frac{\left(k_{1}-k_{Y 1}\right) d}{2 \pi}\right\}_{-}$, where $\{z\}_{+}$ corresponds to the smallest integer greater than $z$, and $\{z\}_{-}$corresponds to the largest integer less than $z$.

Likewise, the reflected wave function $[x \ll-d]$ is given by:

$$
\psi_{R}^{ \pm \vec{K}}(\vec{r})=\sum_{n \in \mathcal{N}} R_{n} e^{i\left(k_{Y 1}^{(n)} y-k_{X 1}^{(n)} x\right)} \sqrt{\frac{k_{1}}{2 v_{F} k_{X 1}^{(n)}}}\left(\mp e^{-i \theta_{\vec{k}_{1}^{(n)}}}\right)_{ \pm \vec{K}}
$$

where $R_{n}$ is the reflection coefficient for the $n^{\text {th }}$ open scattering channel in $\mathcal{N}$. Due to the unitarity condition, $\sum_{n \in \mathcal{N}}\left|R_{n}\right|^{2}+\left|T_{n}\right|^{2}=1$. Expressions for $R_{n}$ and $T_{n}$ are given in Supporting Information.

In Fig. 2, numerical calculations of the total transmission probability, $T_{t o t}=\sum_{n \in \mathcal{N}}\left|T_{n}\right|^{2}$, for $\phi_{i n c}^{ \pm \vec{K}}(\vec{r})$ normally incident $\left[\theta_{\vec{k}_{1}}=0\right]$ to a one-dimensional array of localized cylindrically symmetric scatterers of radius $r_{s}=20 \mathrm{~nm}$ as a function of $\frac{d}{\lambda}$ are shown for the following scattering potentials and lattice spacings: (black) $V_{0}=$ $0.8 \mathrm{eV}$ and $d=150 \mathrm{~nm}$, (green) $V_{0}=0.2 \mathrm{eV}$ and $d=150 \mathrm{~nm}$, and (blue) $V_{0}=-0.9683 \mathrm{eV}$ and $d=156.5 \mathrm{~nm}$. For reference, $T_{\text {tot }}=1$ is represented by a red line, which is the exact transmission probability for a wave normally incident to a constant one-dimensional potential barrier ${ }^{19}$ as shown in Fig. 1(A). For $\frac{d}{\lambda}<1, T_{\text {tot }}=1$ for all $V_{0}$ since only the $n=0$ scattering channel is open, i.e., $\mathcal{N}=\{0\}$. As a result, the incident electron wave is prohibited from direct backscattering due to time-reversal symmetry leading to $T_{t o t}=1$. This can also be understood by the fact that when $\frac{d}{\lambda} \ll 1$, the scattering array effectively appears as a constant one-dimensional potential barrier [Fig. 1(A)] where $T_{\text {tot }}=1$ for $\theta_{\vec{k}_{1}}=0$. However, when $\frac{d}{\lambda} \geq 1$, the incident wave can now backscatter into additional open scattering channels, $k_{X 1}^{(n)}$ and $k_{Y 1}^{(n)}$ for $n \neq 0$ in Eq. (3), that do not correspond to direct backscattering, thereby leading to $T_{t o t} \leq 1$. Although additional open scattering channels are now available for the incident electron wave to scatter into when $\frac{d}{\lambda} \geq 1$ for $\theta_{\vec{k}_{1}}=0, T_{\text {tot }}$ depends on $V_{0}$. For example, $T_{\text {tot }}$ decreased to 0.6277 at $\frac{d}{\lambda}=1.01$ for $V_{0}=0.8 \mathrm{eV}$ and $d=150 \mathrm{~nm}$ [Fig. 2, black curve] and $T_{\text {tot }}=0.0134$ at $\frac{d}{\lambda}=1.1855$ for $V_{0}=-0,9683 \mathrm{eV}$ and $d=31.3 \mathrm{~nm}$ [Fig. 1, blue curve].

At non-normal incidence, the incident Dirac plane wave can undergo specular reflection and therefore have $T_{t o t}<1$. In 

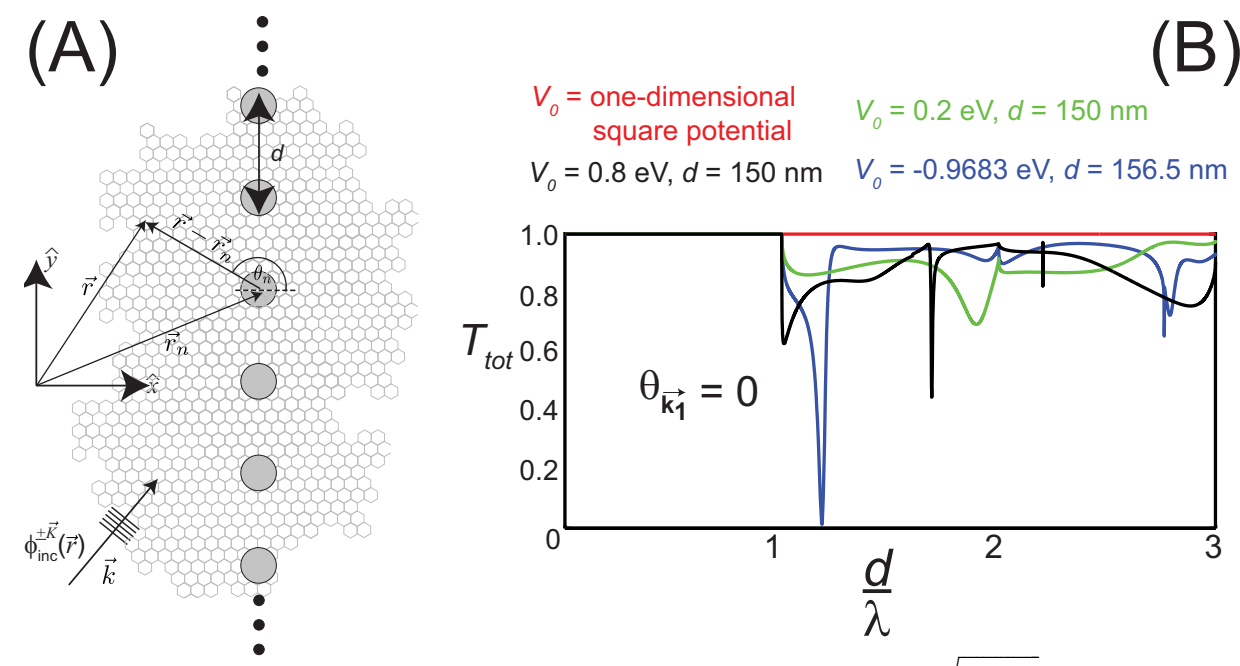

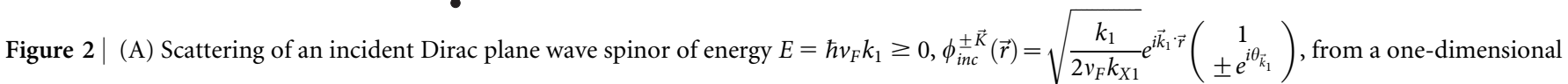
array of localized cylindrically symmetric scatterers in graphene. The unit cell for the scattering array consists of a single scatterer with the position of the $n^{\text {th }}$ scatterer given by $\vec{r}_{n}=n d \hat{y}$. (B) Transmission probability, $T_{t o t}$, for a plane wave normally incident $\left[\theta_{\vec{k}_{1}}=0\right]$ to either a (red line) one-dimensional potential barrier of width $40 \mathrm{~nm}$ [ $T_{\text {tot }}=1$ for all potentials studied in this work] or a one-dimensional array of localized cylindrically symmetric scatterers of radius $r_{s}=20 \mathrm{~nm}$ with the following scattering potentials and lattice spacings: (blue) $V_{0}=-0.9683 \mathrm{eV}$ and $d=156.5 \mathrm{~nm}$, (green) $V_{0}=0.2 \mathrm{eV}$ and $d$ $=150 \mathrm{~nm}$, and (black) $V_{0}=0.8 \mathrm{eV}$ and $d=150 \mathrm{~nm}$. For $\frac{d}{\lambda} \ll 1$, the one-dimensional array of scatterers appear as a uniform one-dimensional potential barrier (black and green) or well (blue) and thus $T_{\text {tot }}=1$. When $\frac{d}{\lambda} \geq 1$, however, the incident electron wave can be reflected into waves with wave vectors $-k_{X 1}^{(n)} \hat{x}+k_{Y 1}^{(n)} \hat{y}$ for $n \neq 0$ that do not correspond to direct backscattering. As a result, $T_{\text {tot }} \leq 1$ when $\frac{d}{\lambda} \geq 1$.

Figure 3, a comparison of $T_{t o t}$ as a function of $\frac{d}{\lambda} \in\left[\frac{\sqrt{2}}{200 \pi}, 3+\frac{\sqrt{2}}{200 \pi}\right]$ and incident angle, $\theta_{\vec{k}_{1}} \in\left[\frac{0.1 \pi}{180}, \frac{89.9 \pi}{180}\right]$, in graphene is shown [Fig. 3, right]. For comparison, the total transmission probability for nonspinor or achiral plane waves found in a regular two-dimensional electron gas (2DEG) with $\hat{H}_{2 D E G}=\frac{\hat{p}^{2}}{2 m}+\hat{V}(x, y)$ is also shown [Fig. 3, left]. The same scattering potentials and lattice spacings used in Figure 2 were also used in the calculations shown in Fig. 3: [Fig. 3(A)] $V_{0}=0.2 \mathrm{eV}$ and $d=150 \mathrm{~nm}$, [Fig. 3(B)] $V_{0}=0.8 \mathrm{eV}$ and $d=150 \mathrm{~nm}$, and [Fig. 3(C)] $V_{0}=-0.9683 \mathrm{eV}$ and $d=$ $156.5 \mathrm{~nm}$. Further details of the calculations in Fig. 3 are given in Supporting Information. For the 2DEG, $T_{\text {tot }}$ was similar for all scattering potentials studied [Fig. 3(A)-3(C), left], with $T_{t o t} \rightarrow 1$ only after $\frac{d}{\lambda} \geq 1$ at $\theta_{\vec{k}_{1}}=0$. For $\frac{d}{\lambda} \ll 1$, there was negligible transmission of the incident wave in the $2 \mathrm{DEG}$ for all $\theta_{\vec{k}_{1}}$. In graphene, however, the dependence of $T_{\text {tot }}$ on $\frac{d}{\lambda}$ and $\theta_{\vec{k}_{1}}$ in Fig. 3 (right) was sensitive to $V_{0}$. For $\frac{d}{\lambda}<1$ and $\theta_{\vec{k}_{1}} \approx 0, T_{\text {tot }} \approx 1$ in all cases as a result of Klein tunneling as previously illustrated in Fig. 2. However, for $\theta_{\vec{k}_{1}} \neq 0$, specular reflection can occur leading to $T_{t o t}<1$ even for $\frac{d}{\lambda}<1$. Again, above $\frac{d}{\lambda} \geq 1$, the incident wave can backscatter along the Bragg directions, thereby leading to a reduction in $T_{\text {tot }}$ even at normal incidence.

In Fig. 3, sharp features in $T_{\text {tot }}$ (indicated by ${ }^{*}$ in Fig. 3 ) were also observed around the following values of $\frac{d}{\lambda}$ in graphene: [Fig. 3(B), right] $\frac{d}{\lambda}=1.6915$ for $V_{0}=0.8 \mathrm{eV}$, [Fig. $3(\mathrm{~A})$, right] $\frac{d}{\lambda}=0.8887$ for $V_{0}=0.2 \mathrm{eV}$, and [Fig. $3(\mathrm{C})$, right] $\frac{d}{\lambda}=1.18725$ for $V_{0}=$ $-0.9683 \mathrm{eV}$. These sharp changes in $T_{\text {tot }}$ appear to result from the interference between partial waves from the individual scatterers at values of $k_{1} d$ where $s_{l} \rightarrow-1$ for at least one of the higher partial waves with $l \geq 2$ while at the same time $\left|s_{0}\right| \in[0.8,1]$ and/or $\left|s_{1}\right| \in[0.8,1]$. Approximate values for these $k_{1} d$ where $s_{l} \rightarrow-1$ can be determined from $s_{l}$ [Eq. (6)] and are solutions to the following equation:

$$
J_{l+1}\left(k_{2} r_{s}\right) Y_{l}\left(k_{1} r_{s}\right)-J_{l}\left(k_{2} r_{s}\right) Y_{l+1}\left(k_{1} r_{s}\right)=0
$$

where $Y_{l}(z)$ is a bessel function of the second-kind. In Figure 3, the interference between the $l=0$ and $l=2$ partial waves was observed in Fig. 3(A) and Fig. 3(C) at $\frac{d}{\lambda}=0.8887\left(\left|s_{0}\right|=0.9355\right.$ and $\left.\left|s_{2}\right|=0.9999\right)$ and $\frac{d}{\lambda}=1.18725\left(\left|s_{0}\right|=0.8863\right.$ and $\left.\left|s_{2}\right|=0.9994\right)$, respectively, whereas the interference between the $l=0, l=1$, and $l=3$ partial waves was observed in Fig. 3(B) at $\frac{d}{\lambda}=1.6915\left(\left|s_{0}\right|=0.7964,\left|s_{1}\right|=\right.$ 0.8005 , and $\left.\left|s_{2}\right|=1\right)$. Note that a similar interference between higher partial waves was also observed in the 2DEG near $\frac{d}{\lambda} \approx 1.50225$ [Fig. 3(C), left] with an attractive scattering potential, $V_{0}=$ $-0.9683 \mathrm{eV}$, which was a result of the interference between the $l$ $=0$ and $l=3$ partial waves $\left(\left|s_{0}\right|=0.9436\right.$ and $\left.\left|s_{3}\right|=0.8609\right)$. Furthermore, calculations of $T_{\text {tot }}$ in the 2DEG using the scattering amplitudes in graphene $\left[s_{l}\right.$ in Eq. (6)] also exhibited sharp features in $T_{\text {tot }}$ at the same values of $k_{1} d$ (data not shown). The effects of partial interference between higher partial waves that suppress forward scattering have been previously noted in graphene ${ }^{20}$ and for Mie scattering in optical systems ${ }^{21}$.

\section{Discussion}

A theory for scattering of electron waves incident to a onedimensional array of localized cylindrically symmetric scatterers [Figure 2(A)] in graphene was used to study the transmission probability through the scattering array as a function of angle of incidence, $\theta_{\vec{k}_{1}}$, and wavelength $\lambda$ [see Supporting Information for a derivation of the theory]. When the spacing between scatterers, $d$, 

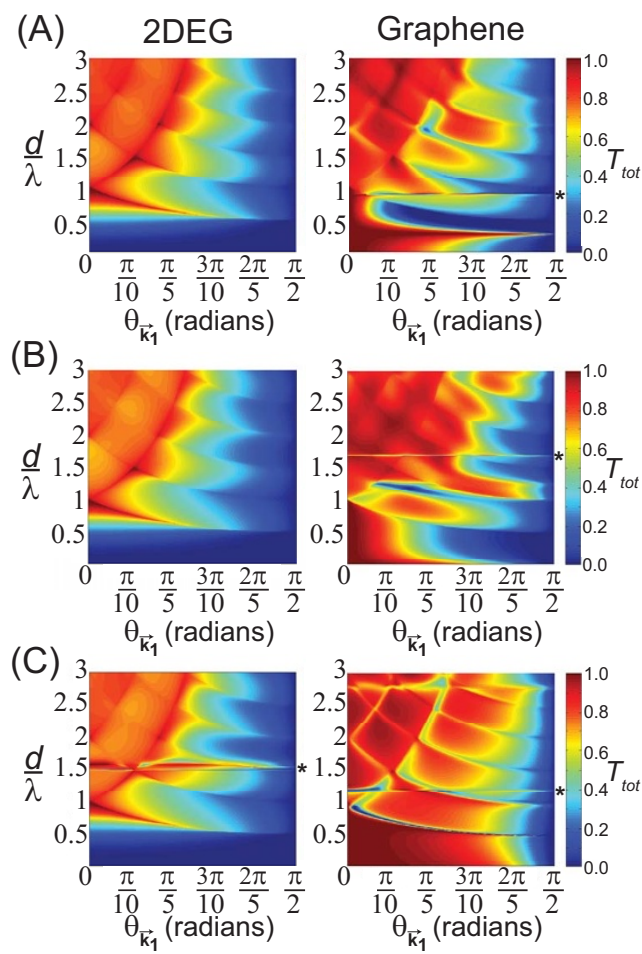

Figure 3 Total transmission probability, $T_{\text {tot }}$, for a plane wave incident to an infinite one-dimensional array of localized cylindrically symmetric scatterers of radius $r_{s}=20 \mathrm{~nm}$ as a function of incident angle,

$\theta_{\vec{k}_{1}} \in\left[\frac{0.1 \pi}{180}, \frac{89.9 \pi}{180}\right]$, and the ratio of lattice spacing to wavelength,

$\frac{d}{\lambda} \in\left[\frac{\sqrt{2}}{200 \pi}, 3+\frac{\sqrt{2}}{200 \pi}\right]$, in a (left) $2 D E G$ and in (right) graphene for

following scattering potentials and lattice spacings: (A) $V_{0}=0.8 \mathrm{eV}\left[I_{\max }\right.$ ranging up to $l_{\max }=4$ in both graphene and the $\left.2 \mathrm{DEG}\right]$ and $d=150 \mathrm{~nm}$, (B) $V_{0}=0.2 \mathrm{eV}\left[I_{\max }\right.$ ranging up to $I_{\max }=4$ in both graphene and the 2DEG] and $d=150 \mathrm{~nm}$, and (C) $V_{0}=-0.9683 \mathrm{eV}\left[I_{\max }\right.$ ranging up to $I_{\max }=6$ for graphene and $I_{\max }=4$ for the $\left.2 \mathrm{DEG}\right]$ and $d=156.5 \mathrm{~nm}$. In Figs. 3(A) and 3(B), the wave vector within the scattering potential was given by $k_{2}=k_{1}-\frac{V_{0}}{\hbar v_{F}}$ in graphene and $-i k_{2}$ in the 2DEG since $k_{1}<\frac{V_{0}}{\hbar v_{F}}$ for the range of $\frac{d}{\lambda}$ plotted in Figs. 3(A) and 3(B). In Fig. 3(C), the wave vector inside the scattering potential was given by $k_{2}=k_{1}+\frac{\left|V_{0}\right|}{\hbar v_{F}}$ in both graphene and the 2DEG. Asterisks $\left(^{*}\right)$ denote those values of $\frac{d}{\lambda}$ where interference between higher partial waves from the individual scatterers generate sharp features in $T_{\text {tot }}$ [Eq. (4)].

is much less than $\lambda\left[\frac{d}{\lambda} \ll 1\right]$, the scattering array in Fig. $2(\mathrm{~A})$ acts like a continuous one-dimensional potential barrier/well [Fig. 1(A)]. In this case, electron waves normally incident to the scattering array are perfectly transmitted as a consequence of Klein tunneling ${ }^{15,19}$. However, when $\frac{d}{\lambda} \geq 1$, the incident electron waves are able to "resolve" the fact that the scattering array is made up of discrete, localized scatterers that can reflect the incident electron wave along the Bragg directions that do not correspond to direct backscattering [Fig. 1(B)]. As a result, the transmission probabilities can be significantly less than one when $\frac{d}{\lambda} \geq 1$, even at normal incidence [Fig. 2]. In effect, placing periodic openings into a constant one-dimensional potential barrier/well can, somewhat counterintuitively, reduce the transmission probability at normal incidence, i.e., suppress Klein tunneling, in graphene. It was demonstrated [Fig. 3, right] that the dependence of the transmission probabilities on incident angle, $\theta_{\vec{k}_{1}}$, and electron wavelength was more sensitive to the scattering potential in graphene relative to that observed for a regular two-dimensional electron gas (2DEG). Furthermore, when $s_{l^{\prime}} \rightarrow-1$ for at least one higher partial wave with $l^{\prime} \geq 2$ while $\left|s_{l^{\prime}}\right| \approx\left|s_{0}\right|$ and/or $\left|s_{l^{\prime}}\right| \approx\left|s_{1}\right|$, the interference between the partial waves from the individual scatterers resulted in sharp features in the transmission probabilities [Fig. 3, right]. Similar features were also observed in the transmission probability for a 2DEG with an attractive scattering potential [Fig. 3(C), left]. While only a one-dimensional periodic array of localized scatterers was considered in this work, any potential that has spatial variations larger than the incident electron wavelength will generate non-specular or diffuse reflection that will suppress Klein tunneling. Such potentials could be useful in realizing future graphene electronic devices, such as a graphene field effect transistor ${ }^{22}$. Finally, the results presented in this work could be applied to other physical systems that behave like massless Dirac fermions, such as the surface states of topological insulators ${ }^{23-25}$, optical analogues of graphene ${ }^{26}$, and trapped ions ${ }^{27}$.

\section{Methods}

The theory for scattering of a massless Dirac plane wave spinor from a onedimensional array of localized cylindrically symmetric scatterers [Fig. 1(B)] is derived in Supporting Information ${ }^{28-32}$, where it is shown that the full scattering solution for $\phi_{i n c}^{ \pm \vec{K}}(\vec{r})$ incident to the scattering array shown in Fig. 1(B), $\psi_{ \pm \vec{K}}(\vec{r})$, can be written as [for $x \neq 0]$ :

$$
\begin{aligned}
& \psi_{ \pm \vec{K}}(\vec{r})=\varphi_{i n c}^{ \pm \vec{K}}+\sum_{l=0}^{l_{\max }} \sum_{n=-\infty}^{\infty} \frac{2 s_{l}}{d} \frac{e^{i\left(k_{Y 1}^{(n)} y+k_{X 1}^{(n)}|x|\right)}}{k_{X 1}^{(n)}}(\operatorname{sign}(x))^{l} \\
& \times\left(\begin{array}{cc}
e^{i \operatorname{sign}(x) l \theta_{\vec{k}_{1}^{(n)}}} & \pm \operatorname{sign}(x) e^{-i \operatorname{sign}(x)(l+1) \theta_{\vec{k}_{1}^{(n)}}} \\
\pm \operatorname{sign}(x) e^{i \operatorname{sign}(x)(l+1) \theta_{\vec{k}_{1}^{(n)}}} & e^{-i \operatorname{sign}(x) l \theta_{\vec{k}_{1}^{(n)}}}
\end{array}\right)_{ \pm \vec{K}} \hat{T}_{l, \pm \vec{K}} \psi_{ \pm \vec{K}}\left(\vec{r}_{0}\right)
\end{aligned}
$$

where $\hat{T}_{l, \pm \vec{K}}$ is the single scatterer $l^{t h}$-partial wave $t$-matrix operator, $l_{\text {max }}$ is the maximum number of partial waves that are included in the calculations,

$k_{Y 1}^{(n)}=k_{Y 1}+\frac{2 \pi n}{d}$ for integer $n$, and either $k_{X 1}^{(n)}=\sqrt{k_{1}^{2}-\left(k_{Y 1}^{(n)}\right)^{2}}$ and

$e^{ \pm i \theta_{\bar{K}_{1}^{(n)}}}=\frac{k_{X 1}^{(n)} \pm i k_{Y 1}^{(n)}}{k_{1}}$ for $k_{1} \geq k_{Y 1}^{(n)}$ or $k_{X 1}^{(n)}=i \sqrt{\left(k_{Y 1}^{(n)}\right)^{2}-k_{1}^{2}}$ and $e^{ \pm i \theta_{\bar{K}_{1}^{(n)}}}=i \frac{k_{X 1}^{(n)} \pm k_{Y 1}^{(n)}}{k_{1}}$

for $k_{1} \leq k_{Y 1}^{(n)}$. In Eq. (5), $\psi_{+\vec{K}}(\vec{r})$ consists of a series of plane waves $[n \in \mathcal{N}]$ that are either transmitted $[x>0]$ or reflected $[x<0]$ from the scattering array along with an infinite number of waves $[n \notin \mathcal{N}]$ that are evanescent along the $\hat{x}$-direction and freely propagating along the $\hat{y}$-direction. These evanescent waves are a consequence of the periodicity of the one-dimensional array of scatterers. The transmission $\left[T_{n}\right.$ in Eq. (2) for $n \in \mathcal{N}]$ and reflection $\left[R_{n}\right.$ in Eq. (3) for $\left.n \in \mathcal{N}\right]$ coefficients can be determined from Eq. (5), and explicit expressions for $T_{n}$ and $R_{n}$ are given in Supporting Information.

In all simulations, each scatterer was modeled as a cylindrically symmetric barrier/ well of potential $V_{0}$ and radius $r_{s}$. For an individual scatterer, the $l^{\text {th }}$ partial wave scattering amplitude is given by ${ }^{28,33}$ :

$$
s_{l}=\frac{J_{l}\left(k_{2} r_{s}\right) J_{l+1}\left(k_{1} r_{s}\right)-J_{l}\left(k_{1} r_{s}\right) J_{l+1}\left(k_{2} r_{s}\right)}{J_{l+1}\left(k_{2} r_{s}\right) H_{l}^{(1)}\left(k_{1} r_{s}\right)-J_{l}\left(k_{2} r_{s}\right) H_{l+1}^{(1)}\left(k_{1} r_{s}\right)}
$$

where $k_{2}=\frac{E-V_{0}}{\hbar v_{F}}$, and $J_{l}(z)$ and $H_{l}^{(1)}(z)$ are first-order bessel and hankel functions of order $l$, respectively. The maximum partial wave used in the calculations, $l_{\max }$, was chosen to take into account $99.9 \%$ of the total scattering amplitude for an individual scatterer, i.e., $\sum_{l=0}^{l_{\max }}\left|s_{l}\right|^{2} \approx 0.999 \sum_{l=0}^{\infty}\left|s_{l}\right|^{2}$. For the calculations of $T_{\text {tot }}$ in a 2DEG [Fig. 3 (left)], previous work ${ }^{32}$ on scattering from one-dimensional periodic grating in a $2 \mathrm{DEG}$ was generalized to include higher partial waves. Details of these calculations are also given in Supporting Information.

1. Novoselov, K. S. et al. Electric field effect in atomically thin carbon films. Science 306, 666-669 (2004).

2. Geim, A. K. \& Novoselov, K. S. The rise of graphene. Nat. Mat. 6, 183-191 (2007).

3. Neto, A. H. C., Guinea, F., Peres, N. M. R., Novosolev, K. S. \& Geim, A. K. The electronic properties of graphene. Rev. Mod. Phys. 81, 109-162 (2009).

4. Wallace, P. R. The band theory of graphite. Phys. Rev. 71, 622-634 (1947).

5. Semenoff, G. W. Condensed-matter simulation of a three-dimensional anomoly. Phys. Rev. Lett. 53, 2449-2452 (1984). 
6. Klein, O. Die reflexion von elektronen an einem potentialsprung nach der relativistischen dynamik von Dirac. Z. Phys. 53, 157-165 (1929).

7. Bena, C. \& Montambaux, G. Remarks on the tight-binding model of graphene. New J. Phys. 11, 095003; DOI:10.1088/1367-2630/11/9/095003 (2009).

8. Zhou, X. et al. Locally altering the electronic properties of graphene by nanoscopically doping it with rhodamine 6G. Nano Lett. 13, 1616-1621 (2013).

9. Bian, S. et al. Covalently patterned graphene surfaces by a force-accelerated DielsAlder reaction. J. Am. Chem. Soc. 135, 9240-9243 (2013).

10. Titov, M., Ostrovsky, P. M., Gornyi, I. V., Schuessler, A. \& Mirlin, A. D. Charge transport in graphene with resonant scatterers. Phys. Rev. Lett. 104, 076802 (2010).

11. Kessler, B. M., Girit, C. O., Zettl, A. \& Bouchiat, V. Tunable superconducting phase transition in metal-decorated graphene sheets. Phys. Rev. Lett. 104, 047001 (2010)

12. Park, C.-H., Yang, L., Son, Y.-W., Cohen, M. L. \& Louie, S. G. Anisotropic behaviours of massless dirac fermions in graphene under periodic potentials. Nat. Phys. 4, 213-217 (2008).

13. Park, C.-H., Son, Y.-W., Yang, L., Cohen, M. L. \& Louie, S. G. Electron beam supercollimation in graphene superlattices. Nano Lett. 8, 2920-2924 (2008).

14. Bai, C. \& Zhang, X. Klein paradox and resonant tunneling in a graphene superlattice. Phys. Rev. B 76, 075430 (2007)

15. Barbier, M., Peeters, F. M., Vasilopoulos, P. \& Pereira, J. M. Dirac and KleinGordon particles in one-dimensional periodic potentials. Phys. Rev. B 77, 115446 (2008).

16. Barbier, M., Vasilopoulos, P. \& Peeters, F. M. Dirac electrons in a Kronig-Penney potential: Dispersion relation and transmission periodic in the strength of the barriers. Phys. Rev. B 80, 205415 (2009).

17. Barbier, M., Vasilopoulos, P. \& Peeters, F. M. Single-layer and bilayer graphene superlattices: collimation, additional Dirac points and Dirac lines. Phil. Trans. $R$. Soc. A 368, 5499-5524 (2010).

18. Pieper, A., Heinisch, R. L., Wellein, G. \& Fehske, H. Dot-bound and dispersive states in graphene quantum dot superlattices. Phys. Rev. B 89, 165121 (2014).

19. Katsnelson, M. I., Novoselov, K. S. \& Geim, A. K. Chiral tunnelling and the Klein paradox in graphene. Nat. Phys. 2, 620-625 (2006).

20. Heinisch, R. L., Bronold, F. X. \& Fehske, H. Mie scattering analog in graphene: Lensing, particle confinement, and depletion of Klein tunneling. Phys. Rev. B 87, 155409 (2013).

21. Lock, J. A. Cooperative effects among partial waves in mie scattering. J. Opt. Soc. Am. A 5, 2032-2044 (1988).

22. Jang, M. S., Kim, H., Son, Y.-W., Atwater, H. A. \& Goddard III, W. A. Graphene field effect transistor without an energy gap. Proc. Nat. Acad. Sci 110, 8786-8789 (2013).

23. Zhang, T. et al. Experimental demonstration of topological surface states protected by time-reversal symmetry. Phys. Rev. Lett. 103, 266803.

24. Hsieh, D. et al. Observation of time-reversal-protected single-Dirac-cone topological-insulator states in $\mathrm{Bi}_{2} \mathrm{Te}_{3}$ and $\mathrm{Sb}_{2} \mathrm{Te}_{3}$. Phys. Rev. Lett. 103, 146401 (2009).

25. Hasan, M. Z. \& Kane, C. L. Topological insulators. Rev. Mod. Phys. 82, 3045 $(2010)$
26. Zhu, S. L., Wang, B. \& Duan, L. M. Simulation and detection of Dirac fermions with cold atoms in an optical lattice. Phys. Rev. Lett. 98, 260402 (2007).

27. Gerritsma, R. et al. Quantum simulation of the Klein paradox with trapped ions. Phys. Rev. Lett. 106, 060503 (2011).

28. Vaishnav, J. Y., Anderson, J. Q. \& Walls, J. D. Intravalley multiple scattering of quasiparticles in graphene. Phys. Rev. B 83, 165437 (2011)

29. Yasumoto, K. \& Yoshitomi, K. Efficient calculation of lattice sums for free-space periodic Green's function. IEEE Trans. Antennas Propagat. 47, 1050-1055 (1999).

30. Nicorovici, N. A., McPhedran, R. C. \& Petit, R. Efficient calculation of the Green's function for electromagnetic scattering by gratings. Phys. Rev. E 49, 4563-4577 (1994).

31. Pereira, J. M., Mlinar, V., Peeters, F. M. \& Vasilopoulos, P. Confined states and direction-dependent transmission in graphene quantum wells. Phys. Rev. B 74, 045424 (2006).

32. Vaishnav, J. Y., Walls, J. D., Apratim, M. \& Heller, E. J. Matter-wave scattering and guiding by atomic arrays. Phys. Rev. A 76, 013620 (2007).

33. Katsnelson, M. I. \& Novoselov, K. S. Graphene: New bridge between condensed matter physics and quantum electrodynamics. Sol. State Comm. 143, 3-13 (2007)

\section{Acknowledgments}

We thank Dr. J. Y. Vaishnav for comments on the manuscript. This work was supported by the National Science Foundation under CHE - 1056846 and from funds from the University of Miami.

\section{Author contributions}

J.D.W. performed theoretical and numerical calculations and wrote manuscript. D.H. worked on initial simulations for the project and helped in manuscript preparation.

\section{Additional information}

Supplementary information accompanies this paper at http://www.nature.com/ scientificreports

Competing financial interests: The authors declare no competing financial interests.

How to cite this article: Walls, J.D. \& Hadad, D. Suppressing Klein tunneling in graphene using a one-dimensional array of localized scatterers. Sci. Rep. 5, 8435; DOI:10.1038/ srep08435 (2015)

This work is licensed under a Creative Commons Attribution 4.0 International License. The images or other third party material in this article are included in the article's Creative Commons license, unless indicated otherwise in the credit line; if the material is not included under the Creative Commons license, users will need to obtain permission from the license holder in order to reproduce the material. To view a copy of this license, visit http://creativecommons.org/licenses/by/4.0/ 